\title{
Stable Keypoint Recognition using Viewpoint Generative Learning
}

\author{
Takumi Yoshida ${ }^{1}$, Hideo Saito ${ }^{1}$, Masayoshi Shimizu ${ }^{2}$ and Akinori Taguchi ${ }^{2}$ \\ ${ }^{1}$ Keio University, Minato, Tokyo, Japan \\ ${ }^{2}$ Fujitsu Laboratories, Kawasaki, Japan \\ $\{$ tkm, saito\}@hvrl.ics.keio.ac.jp, \{shimizu.masa,taguchi.akinori\}@jp.fujitsu.com
}

Keywords: Generative Learning, Keypoint Recognition, Local Features, Pose Estimation.

\begin{abstract}
We propose a stable keypoint recognition method that is robust to viewpoint changes. Conventional local features such as SIFT, SURF, etc., have scale and rotation invariance but often fail in matching points when the camera pose significantly changes. In order to solve this problem, we adopt viewpoint generative learning. By generating various patterns as seen from different viewpoints and collecting local invariant features, our system can learn feature descriptors under various camera poses for each keypoint before actual matching. Experimental results comparing usual local feature matching or patch classification method show both robustness and fastness of learning.
\end{abstract}

\section{INTRODUCTION}

Matching between two images by finding points of correspondence is an important issue in Computer Vision. In particular, object detection and tracking for AR or SLAM require robustness to camera movements, which has been recently achieved by local features. To recognize keypoints accurately, the detector with high repeatability and the descriptor with high precision are necessary; the keypoint is detected at the same location in various conditions of the target and it has static value even in various conditions.

To solve this matching problem, local invariant features have been proposed. As a prior region detector research, combining either traditional Harris or Hessian corner detectors followed by Laplacian scale selection and affine adaption were investigated by (Baumberg, 2000), (Mikolajczyk and Schmid, 2004) and (Mikolajczyk et al., 2005). SIFT (Lowe, 2004) is the pioneer of the high performance feature descriptors. This detector is based on a scale space with Differences of Gaussians and its descriptor is highly valued for the scale and rotation invariance based on the evaluation research (Mikolajczyk and Schmid, 2005). After SIFT appeared, many researches related to SIFT has extended its algorithm. Interest Point Groups (Brown and Lowe, 2002) introduces a family of feature descriptors which use groups of keypoints. This is based on an approach that each match implies a hypothesis of the local 2D transformation. By choosing groups of a few keypoints and using them to de- fine a local coordinate frame, this feature descriptors which are invariant under similarities, affinities and homographies can be formed. GLOH (Mikolajczyk and Schmid, 2005) computes a SIFT-like descriptor for a log-polar location grid using PCA for data compression. Some modified log-polar descriptors aim to be smart for orientation invariance and light-weight computation (Bellavia et al., 2010) (Takacs et al., 2010). SURF (Bay et al., 2006) is designed to have faster computation than SIFT without degrading its performance. The Haar wavelet approximation of the blob detector based on the Hessian determinant is efficiently computed at different scales using integral images. The motivation of CenSurE detector (Agrawal et al., 2008) is to execute a full spatial resolution in a multiscale detector while SIFT and SURF detectors have just subsampled resolutions.

However, the invariance of these conventional local features is limited to only scale and rotation changes; they are sensitive to camera movements like tilting. In this paper, we propose a stable keypoint recognition method using viewpoint generative learning that achieves robustness to camera movements. By generating various patterns as seen from different viewpoints and collecting feature descriptors, our system can learn a set of different descriptors under various camera poses before actual matching. Our learning can achieve higher repeatability and precision while maximizing each local feature's inherent advantage. Furthermore, our learning takes less than half a minute due to clustering of the descriptors. 


\section{RELATED WORKS}

ASIFT (Morel and Yu, 2009) is an alternative approach following SIFT that was developed for whole affine invariance by introducing two parameters defining the longitude and latitude angle that is equivalent to the tilt in the camera affine model. ASIFT gains impressive precision but this algorithm makes matching several times slower than SIFT (2.25 times in the work of (Morel and Yu, 2009)).

On the other hand, Randomized Trees (Lepetit and Fua, 2006) and Random Ferns (Ozuysal et al., 2009) casted a keypoint recognition problem as a patch classification problem. This approach relies on an offline learning in which the patches of the different views are used to train trees. It recognizes the patches on the basis of a few pairwise intensity comparisons, so this method achieves fast run-time performance. Our proposed viewpoint generative learning (VGL) is inspired by a learning technique for randomized trees base classification (RTs). However, there are two big differences between VGL and RTs:

1) The keypoints selection of RTs is limited to appearances on the reference image. VGL selects a stable keypoint, which are not only found in the reference image but also in the generated patterns. Thus, RTs uses stable keypoints for narrowing the focus but VGL can improve the repeatability of a detector.

2) To search matched keypoints, RTs simply checks the intensity in the local patch, which demands many patch transformations and generations in order to be robust to viewpoint changes. For fast recognition this is one of the contributions, but we also think recent local features would be more effective for robustness of matching and speed of learning. Because they include invariance with their own algorithm, we just have to generate a few dozen patterns. This leads to the advantage that VGL is much faster than RTs for offline learning.

\section{VIEWPOINT GENERATIVE LEARNING}

Some local features have scale and rotation invariance due to their own algorithm. Our approach is to collect these invariant features on the generated patterns as seen from different viewpoints. Viewpoint generative learning enables us to train with various data without actually collecting them. As long as we use a local feature to detect or recognize a target, only one reference image is needed to learn. Therefore, for offline learning, we generate various patterns, extract stable keypoints from them and create a database of collected features. After learning, the reference image and an input image can be matched by comparing them using the database.

\subsection{Generation of Various Patterns}

First, we generate various patterns as seen from different viewpoints that are computed from one reference image of the target. We apply not an affine transformation but perspective transformation that reflects more actual camera pose changes. The viewpoint model and the rotation matrix $\mathbf{R}$ are shown in Figure 1 and Equation 1. The angles $\phi$ and $\theta$ are the camera optical axis longitude and latitude. The angle $\psi$ parameterizes the camera spin.

$\mathbf{R}=\left[\begin{array}{ccc}\cos \psi & \sin \psi & 0 \\ -\sin \psi & \cos \psi & 0 \\ 0 & 0 & 1\end{array}\right]\left[\begin{array}{ccc}\cos \phi & 0 & \sin \phi \\ 0 & 1 & 0 \\ -\sin \phi & 0 & \cos \phi\end{array}\right]\left[\begin{array}{ccc}1 & 0 & 0 \\ 0 & \cos \theta & \sin \theta \\ 0 & -\sin \theta & \cos \theta\end{array}\right]$

The rotation ranges are set as follows $\phi \in$ $\left[-75^{\circ}, 75^{\circ}\right], \theta \in\left[-75^{\circ}, 75^{\circ}\right]$. In this research we use rotation invariant features, so that the camera spin $\psi$ does not move. To calculate perspective transformation matrix $\mathbf{P}$, we obtain an intrinsic camera parameter matrix $\mathbf{A}$ and an extrinsic parameter, the distance $d$ to a target plane. Moreover, due to scale invariant features, the distance $d$ is fixed by choosing focal length. Therefore, we have $\mathbf{A}, \mathbf{R}$ and translation matrix $t=(0,0, d)^{T}$ for $\mathbf{P}=\mathbf{A}(\mathbf{R} \mid t)$.

\subsection{Selection of Stable Keypoints}

On each generated pattern, we detect keypoints by using a local feature detector. To increase repeatability, we select the stable keypoints that have high detectability, defined as how often the same keypoint is detected in different pose patterns, at stable locations. Because we know the transformation matrix to generate the patterns, we can also consider those points to be the same keypoint by projection and calculation of distance. If no keypoint is detected around the projected position of the stable keypoint, we conveniently create a keypoint at that position on the reference image. As presented above, this is one of the advantages of our proposed method: being able to cover new keypoints found in generated patterns with large pose change that were not detected in the reference image. Figure 2 shows the example of a stable keypoint creation.

Since all generated patterns are processed, we select the keypoints with the highest rank in terms of detectability as stable keypoints. In our experiments, the number of stable keypoints is decided by an association with the feature database creation in Sec. 3.3. 


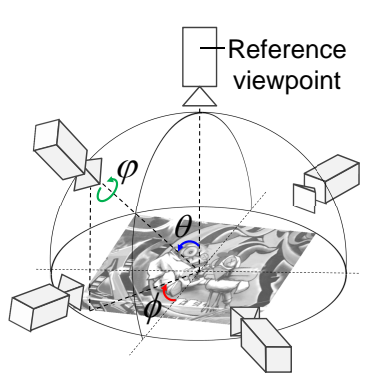

Figure 1: This hemisphere represents the model of viewpoints.

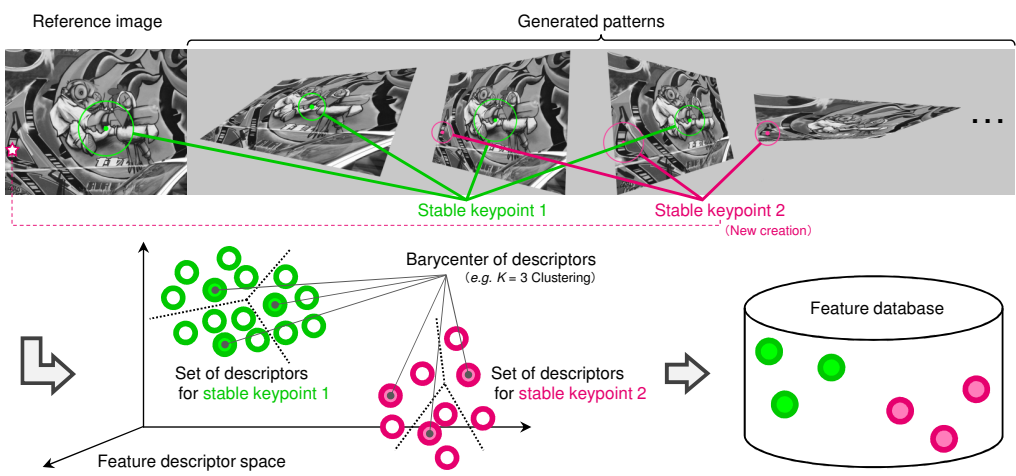

Figure 2: The database includes the barycenter of the set of descriptors for each stable keypoint by using k-means clustering method.

\subsection{Database of Feature Descriptors}

As a result of selecting stable keypoints, the number of descriptors for each keypoint is the number of patterns were that keypoint was detected. However, it would be inefficient for finding correspondence to create feature database by adding all similar descriptors. To avoid this inefficiency, we need to create clusters of descriptors. Famous k-means algorithm begins with $k$ arbitrary barycenter, typically chosen uniformly at random from the data. k-means++ (Arthur and Vassilvitskii, 2007) offers a way of initializing k-means by choosing random starting centers with very specific probabilities. Therefore, k-means++ converge in few iterations, and we can quickly finish learning by using the barycenter of clusters as descriptors.

Figure 2 illustrates the overview of database creation. It is important that we cluster the descriptor collection of each stable keypoint. As a result, the features in the database directly connect with the stable keypoint. When the number of stable keypoints is $N$ and the cluster is $K$, we create $N \times K$ feature descriptors in the database.

\section{KEYPOINT RECOGNITION AND POSE ESTIMATION}

In online phase, we detect keypoints and describe descriptors by using local features on an input image. Then we compare the obtained descriptors with features in the generated database by computing Euclidean distance. To decrease false matching, we apply nearest-neighbor distance ratio (Mikolajczyk et al., 2005) to the distance ratio between the first and the second. The keypoints are matched when the first distance is less than $\tau$ times of the second. The threshold $\tau$ is empirically set to 0.5 in all experiments. Moreover, to remove outliers, we use a robust estimator RANSAC and then compute homography via the Levenberg-Marquardt method.

\section{EXPERIMENTAL RESULT}

Mikolajczyk et al. performed repeatability and precision test of different local features, and provided the dataset with corresponding ground-truth (Mikolajczyk and Schmid, 2005). We used the two viewpoint change sequences in their dataset ('Graffiti' and 'Wall') for tuning the parameters of our method. Each sequence contains a reference view, and five test views at $20^{\circ}, 30^{\circ}, 40^{\circ}, 50^{\circ}$, and $60^{\circ}$ angles numbered from 1 to 5 . As the performance criteria, we use the number of correct matches and the precision:

$$
\text { precision }=\frac{\text { correct matches }}{\text { correct matches }+ \text { false matches }}
$$

Correct matches are determined by the Euclidean distance between a matched keypoint position on a test image and a projected position computed from the other matched keypoint on the reference image by using ground truth homography. When the distance is below a threshold ( 2 pixels), the matching is accepted as correct match in this all experiments.

In addition we introduce an evaluation measure (Lieberknecht et al., 2009) that performs pose estimation. The precision is a meaningful criterion with which we can compare the different local feature descriptors. However, in the case of pose estimation, high precision does not necessarily correspond to accurate estimation. This evaluation is based on four reference points placed on the reference images. We have a ground truth homography and an estimated one, so that points are projected to the ground truth 
point $p_{j}$ and the estimated point $q_{j}$. The RMS distance err is computed as:

$$
e r r=\sqrt{\frac{1}{4} \sum_{j=1}^{4}\left\|p_{j}-q_{j}\right\|^{2}}
$$

As we regard the higher RMS error as a sign to detect algorithm failure, we remove the pixels where err is greater than 10 . We firstly used that precision evaluation to find the best values for our different parameters. Then, we compared our results with the ones of several different local features. Finally, we showed the difference in performances of the proposed method and a patch classification method.

\subsection{Parameter Selection}

We can change three main parameters for viewpoint generative learning. One is the number of generated patterns $W$. Because the rotation ranges are set to $\phi, \theta \in\left[-75^{\circ}, 75^{\circ}\right]$, the interval angle affects the number of patterns $W$ that we will have to generate. For example, the combinations of interval angle $\phi$, interval angle $\theta$ and $W$ are $\left(30^{\circ}, 30^{\circ}, 36\right),\left(25^{\circ}, 25^{\circ}, 49\right)$, $\left(15^{\circ}, 15^{\circ}, 121\right),\left(10^{\circ}, 10^{\circ}, 256\right)$ and $\left(5^{\circ}, 5^{\circ}, 961\right)$. Of course the interval angles $\phi$ and $\theta$ are respectively changed, so that we can test 25 combinations.

Another is the number of stable keypoints $N$. The other is the number of clusters $K$ for k-means++. To compare performance variation, we evaluated precision and learning time by using Graffiti and Wall sequences with SIFT. In particular, the performances in terms of precision and computation time in case of large viewpoint change are a really important point. Thus, for deciding these three parameters, the precision is performed about Nos. 3, 4 and 5 views to give more impact to the case of large viewpoint change. Then because SIFT could detect 4654 keypoints on the reference image of Graffiti and 3393 keypoints on the reference image of Wall, we changed the number of stable keypoint from 500 to 6500 . The learning time is measured from inputting the reference image to the end of the creation of the database of feature descriptors. All the experiments work on Intel Core i3 $3.07 \mathrm{GHz}$ CPU, 2.92 GB RAM and GeForce 310 $589 \mathrm{MHz}$ GPU.

The results are shown in Figure 3 and Table 1. The precision changes for clusters and stable keypoints are presented in Figure 3(a) and Figure 3(b). The more clusters there are, the greater the precision is. However, in the case of more than seven clusters, there is little or no increase in the precision. More stable keypoints also leads to higher precision but in the case of over the half number of the reference keypoints, the precision has little increasing. Incidentally, this relationship does not affect learning time thanks to kmeans quickness. On the other hand, the number of generated patterns directly affects the learning time (Table 1). To evaluate this in an integrated way, we use the precision average of the viewpoint change sequences. According to Figure 3(c), even if the number of pattern increases, this does not mean that the accuracy improves. To be fair trade-off between accuracy and learning speed, we have accepted the following parameter: 7 for $\mathrm{k}$-means $K, 30^{\circ}$ for interval angle $\phi$ and $25^{\circ}$ for interval angle $\theta$. As a result, we generate 42 patterns that take 14 seconds about Graffiti or 25 seconds about Wall.

Table 1: The learning time (sec.) by changing the number of patterns.

\begin{tabular}{c|c|c|c|c|c}
\hline Number of patterns & 36 & 49 & 121 & 256 & 961 \\
\hline \hline Learning time (Graf) & 12 & 16 & 49 & 118 & 611 \\
\hline Learning time (Wall) & 21 & 30 & 92 & 241 & 1680 \\
\hline
\end{tabular}

As described in Sec. 3.3, the number of features in the database is represented as $N \times K$. When $N \times K$ is lower than the number of the reference keypoints, matching run-time is equal to or greater than a default use. Therefore, for real-time requirement without accuracy degradation, we should make consideration of the parameter $N$ and $K$.

\subsection{Test Local Features}

The proposed viewpoint generative learning (VGL) can be adopted for any local features including keypoint detector and descriptor. We used SIFT implemented in SiftGPU (www.cs.unc.edu/ $\sim \mathrm{ccwu} / \mathrm{siftgpu} /$ ), SURF and CenSurE (STAR) implemented in OpenCV (opencv.willowgarage.com), M-SURF (Modified-SURF) descriptor implemented in OpenSURF (www.chrisevansdev.com/computervision-opensurf.html) for combination use with CenSurE, Harris/Hessian - Laplacian and Harris/Hessian - Affine with GLOH we used the implementation proposed by the dataset provider (www.robots.ox.ac.uk/ $\sim v g g /$ research/affine/). To compare fairly, the same parameters are set to extract these local features for all the different approaches. And also we use the same number of stable keypoints as the number of reference keypoints. The setting of the other parameters for learning is performed as described in Sec. 5.1.

Figure 4 shows the precision and Table 2 shows the RMS error. The higher the precision is the better; the lower the RMS error is the better. Comparing with default feature matching, VGL improves the precision and achieves the accurate pose estimation in most cases. In particular, in Nos. 4 and 5 Graffiti 


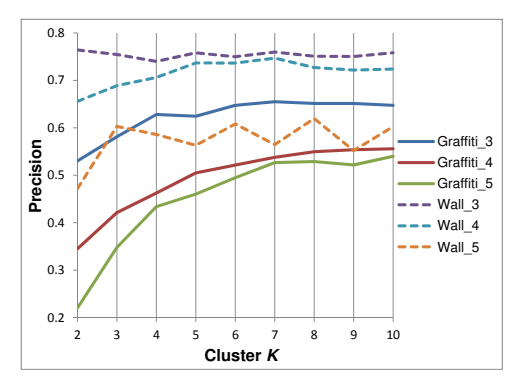

(a) Precision test for the num of cluster K.

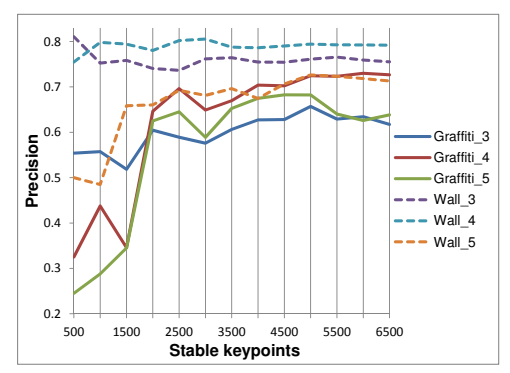

(b) Precision test for the num of stable keypoints.

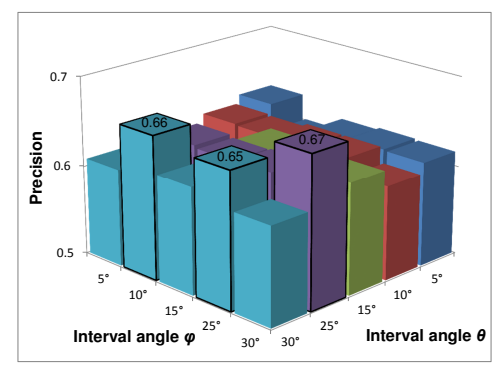

(c) Precision test for the num of patterns.

Figure 3: The choice of most effective parameter for viewpoint generative learning.

cases, the default method can neither find points of correspondence nor estimate that pose. Even in such a difficult viewpoint change scene, the proposed VGL can recognize keypoints and accurately get points of correspondence. Additionally in the challenging situation like Nos. 4 and 5 Wall, the proposed method also outperforms the default one. The reason why about No. 3 Wall the default method can more correct matches is because the image texture does not change from reference texture. In such case, a keypoint with only reference information performs better than a keypoint with several feature descriptors.

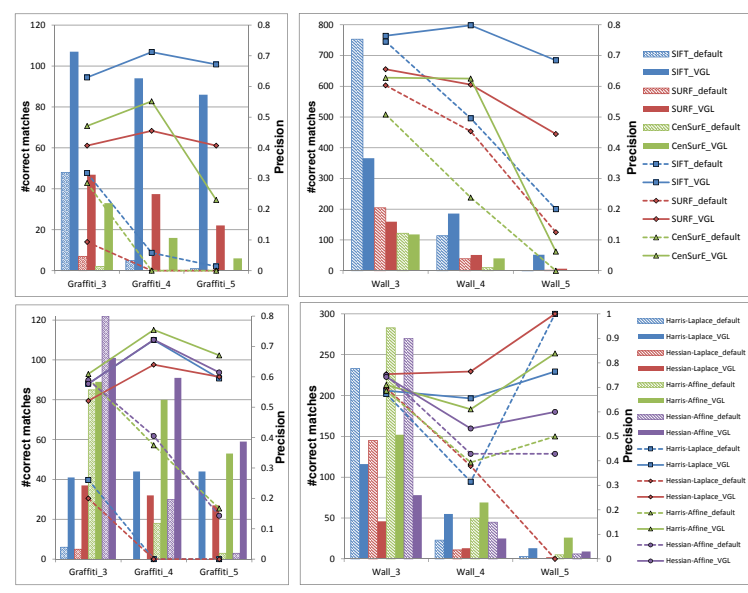

Figure 4: The comparison of precision by using some local features.

\subsection{Comparison with ASIFT and Ferns}

Figures 5 and 6 are the results of comparing with ASIFT and Random Ferns. As they are robust to viewpoint changes, we add the other dataset ('Adam' and 'Magazine') with over $80^{\circ}$ angle view. ASIFT and the test images are provided by the authors (www.cmap.polytechnique.fr/ yu/research/ASIFT/) and Ferns is implemented in OpenCV. The learning time and accuracy of Ferns depend on the number of generated patterns. We have tested various numbers
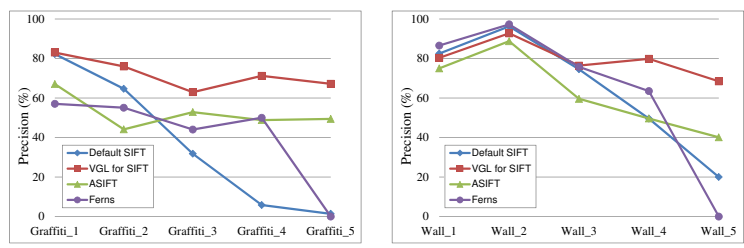

Figure 5: The comparison with related approach.

Table 2: The RMS error in Graffiti (G) and Wall (W). Default is in top row and VGL is in bottom row.

\begin{tabular}{c|c|c|c|c|c|c|c}
\hline & SIFT & SURF & CenSurE & Har-Lap & Hes-Lap & Har-Aff & Hes-Aff \\
\hline \hline G 3 & 2.15 & 8.18 & - & - & 7.28 & 4.18 & 3.45 \\
& 1.21 & 1.84 & 4.47 & 2.69 & 4.69 & 2.45 & 3.01 \\
\hline G 4 & - & - & - & - & - & 4.82 & 6.01 \\
& 2.92 & 5.81 & 7.19 & 5.14 & 4.05 & 4.89 & 2.66 \\
\hline G 5 & - & - & - & - & - & - & 8.08 \\
& 2.77 & 5.44 & - & 5.60 & 6.51 & 4.48 & 5.71 \\
\hline \hline W 3 & 6.34 & 5.98 & 6.01 & 5.85 & 6.55 & 7.29 & 6.14 \\
& 5.96 & 5.46 & 5.73 & 5.18 & 5.17 & 6.03 & 5.96 \\
\hline W 4 & 7.71 & 9.09 & 8.98 & 9.35 & 9.07 & 9.01 & 9.41 \\
& 8.77 & 6.36 & 6.45 & 9.78 & 8.14 & 8.78 & 7.45 \\
\hline W 5 & 7.44 & - & - & - & - & - & - \\
& 6.48 & 5.00 & - & 3.59 & - & 6.24 & - \\
\hline \hline
\end{tabular}

of patterns and found that 5000 patterns performed the best accuracy in No. 4 test.

ASIFT can get most points of correspondence but its precision is not best in this experiment. In terms of processing time, ASIFT takes 47 seconds per image but SIFT_VGL takes 3 seconds about Graffiti. Therefore, ASIFT is not suitable for fast run-time purpose, while VGL can ensure the faster computation with higher accuracy.

On the other hand, Ferns generally performs much faster than local features matching method but it is not very robust to camera movements. Its learning also takes a long time due to huge patch training; more than 10 minutes about Graffiti. In contrast, VGL performs highly accurately with short learning time (less than 15 seconds). Thanks to many widely spread correspondences of stable keypoints, VGL can also correctly estimate a target pose even in the case of a large viewpoint change. Meanwhile local features applying VGL work until $80^{\circ}$ angle changes with fast learning, less than quarter of a minute. 


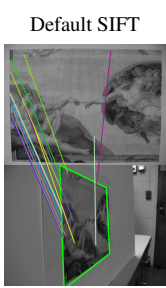

$14 / 33(42 \%)$

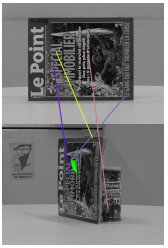

$0 / 51(0 \%)$

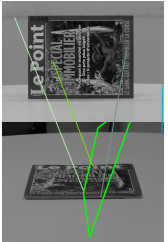

$0 / 12(0 \%)$

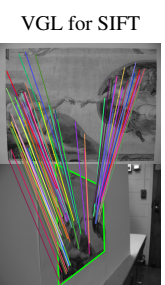

$83 / 111(75 \%)$

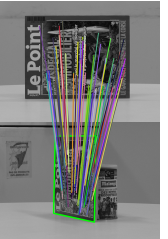

$81 / 128(63 \%)$

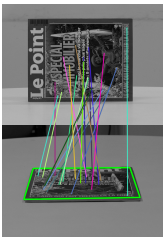

$24 / 37(65 \%)$
ASIFT

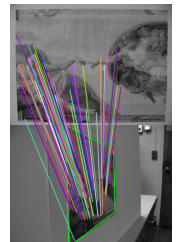

$80 / 149(54 \%)$

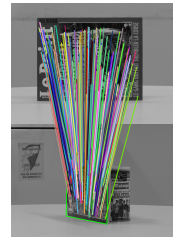

$245 / 437(56 \%)$

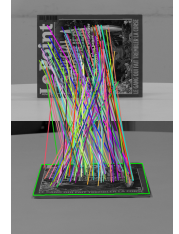

$125 / 235(53 \%)$
Ferns

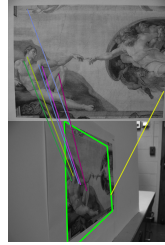

$7 / 10(70 \%)$

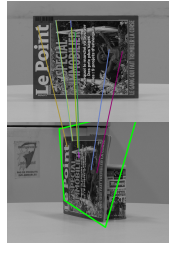

$8 / 16(50 \%)$

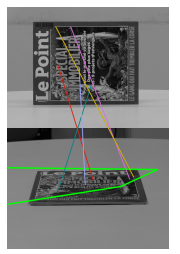

$2 / 10(20 \%)$
Figure 6: The keypoints of correspondence and the pose estimation result in Adam and Magazine. These numbers show the correct matches / all matches.

\section{CONCLUSIONS}

We have presented a stable keypoint recognition that is robust to viewpoint changes. By generating various patterns as seen from different viewpoints and the clusterization of the collected local invariant features, our system learns a set of descriptors under various camera poses for each keypoints before actual matching. This learning achieves more repeatability and precision while maximizing each local feature's inherent advantage, and takes less than quarter of a minute.

Recent years the evaluation research for the combination of local feature detector and descriptor have been done (Gauglitz et al., 2011). As any local feature descriptor algorithm that is described as a high dimensional value can be applied to our framework, in the case of feature with low scale or rotation invariance, we can still apply our method after generating a new viewpoint pattern to cover that situation.

\section{REFERENCES}

Agrawal, M., Konolige, K., and Blas, M. R. (2008). Censure: Center surround extremas for realtime feature detection and matching. ECCV, 5305:102-115.

Arthur, D. and Vassilvitskii, S. (2007). k-means++: The advantages of careful seeding. Proceedings of the eighteenth annual ACM-SIAM symposium on Discrete algorithms, pages 1027-1035.

Baumberg, A. (2000). Reliable feature matching across widely separated views. CVPR, pages 774-781.

Bay, H., Tuytelaars, T., Gool, V., and L. (2006). Surf Speeded up robust features. ECCV, 3951:404-417.

Bellavia, F., Tegolo, D., and Trucco, E. (2010). Improving sift-based descriptors stability to rotations. ICPR, pages $3460-3463$

Brown, M. and Lowe, D. (2002). Invariant features from interest point groups. $B M V C$, pages 656-665.

Gauglitz, S., Hollerer, T., and Turk, M. (2011). Evaluation of interest point detectors and feature descriptors for visual tracking. IJCV, 94:335-360.

Lepetit, V. and Fua, P. (2006). Keypoint recognition using randomized trees. IEEE Transactions on Pattern Analysis and Machine Intelligence, 28(9):1465-1479.

Lieberknecht, S., Benhimane, S., Meier, P., and Navab, N. (2009). A dataset and evaluation methodology for template-based tracking algorithms. ISMAR, pages $145-151$

Lowe, D. G. (2004). Distinctive image features from scaleinvariant keypoints. IJCV, 60:91-110.

Mikolajczyk, K. and Schmid, C. (2004). Scale \& affine invariant interest point detectors. IJCV, 60:63-86.

Mikolajczyk, K. and Schmid, C. (2005). A performance evaluation of local descriptors. IEEE Transactions on Pattern Analysis and Machine Intelligence, 27:16151630.

Mikolajczyk, K., Tuytelaars, T., Schmid, C., Zisserman, A., Matas, J., Schaffalitzky, F., Kadir, T., and Van Gool, L. (2005). A comparison of affine region detectors. IJCV , 65:43-72.

Morel, J. M. and Yu, G. (2009). Asift: A new framework for fully affine invariant image comparison. SIAM Journal on Imaging Sciences, 2(2):438-469.

Ozuysal, M., Calonder, M., Lepetit, V., and Fua, P. (2009). Fast keypoint recognition using random ferns. IEEE Transactions on Pattern Analysis and Machine Intelligence, 32(3):448-461.

Takacs, G., Chandrasekhar, V., Chen, H., Chen, D., Tsai, S., Grzeszczuk, R., and Girod, B. (2010). Permutable descriptors for orientation-invariant image matching. SPIE. 